\title{
Simulation of Fretting Fatigue in Conformal and Concentrated Contacts
}

\author{
Dag Fritzson ${ }^{1)}$, Jonas Ståhl ${ }^{1{ }^{*}}$ and Guillermo Morales-Espejel ${ }^{2)}$ \\ ${ }^{1)}$ SKF Engineering \& Research Centre \\ MDC, RKs-2, SE-415 50 GÖTEBORG, Sweden \\ ${ }^{2}$ SKF Engineering \& Research Centre \\ P.O. Box 2350, 3430 DT NIEUWEGEIN, The Netherlands \\ "Corresponding author: jonas.stahl@skf.com
}

( Manuscript received 19 December 2009; accepted 2 April I 2010; published 31 January 2011 )

(Presented at the World Tribology Congress 2009, Kyoto, 6-11 September, 2009)

\begin{abstract}
Fretting fatigue is a possible failure mode of rolling bearings, as well as for other machine elements with contacts. For a rotating bearing it normally can occur in the interfaces between outer ring/housing, and inner ring/shaft. For a non-rotating bearing subjected to small motion due to vibration, it can occur in the concentrated rolling element/ring contacts as well (mainly related to false brinelling). Detailed models for fretting-fatigue have been transferred and adapted to a bearing simulation framework called BEAring Simulation Toolbox (BEAST). It allows the use of the fretting-fatigue model to predict failures and be used by engineers in developing new solutions. Another important usage is to provide a platform for further development of other fretting-fatigue models, fretting-wear and false brinelling models. The results from two different examples are presented; one model with an oscillating body in contact with another, representing a concentrated contact, and one model with a Deep Groove Ball Bearing (DGBB) mounted inside a ring-like housing. Results are presented in terms of contact pressures, shear forces at the surfaces and a parameter representing the fretting risk according to the Dang-Van criterion. The model for the concentrated contact is used to verify the simulations against stand-alone computer code and experiments. The DGBB model shows how the fretting-fatigue related results are influenced by the ball pass frequency and the flexibility of the bearing and the housing.
\end{abstract}

Keywords: fretting-fatigue, dynamic simulations, rolling bearings, Dang-Van

\section{Introduction}

Fretting damage occurs between two normally loaded components that are also subject to small tangential oscillations. This complex phenomenon involves mechanical aspects such as adhesive and/or abrasive wear and crack formation as well as chemical aspects such as oxidation. It is observed in numerous mechanical components including spline couplings, wire ropes, dove tail joints, hubs or discs press fitted on to rotating shafts etc. In bearing applications it frequently occurs at the interfaces of the inner ring and the shaft, outer ring and housing and the rolling element and the race. In the latter case, it is often referred to as false brinelling.

Two types of damage are associated with fretting contacts: fretting wear, which occurs at greater amplitudes of oscillation when gross slip between contacting components takes place, and fretting fatigue occurring at very small oscillatory amplitudes when only incipient sliding is present i.e. the contact area includes both, stick and slip zones. Figure 1 schematically illustrates when each type of damage may be prevalent while Fig. 2 is a qualitative example that shows a scar from a typical ball-on-flat fretting contact operating in the incipient sliding regime. The inner stick zone and the slip annulus are clearly visible in this photograph as is the presence of a micro-crack.

Commercial finite element or multi-body dynamics tools are not capable to efficiently handle the contact situations. The dynamic simulation tool BEAST ${ }^{1}$, developed internally, is a multi-body simulation tool specialized in detailed contact calculations, making it a very efficient tool for rolling bearings and other machine elements where contacts are important. The model simultaneously solves the transient multi-body equations, the structural deformations, the thermal balance, and the local lubricated contact conditions (if 
any in the fretting contact). The tool is used for optimizing the product design, for evaluating performance under various application conditions, and for advanced failure analysis.

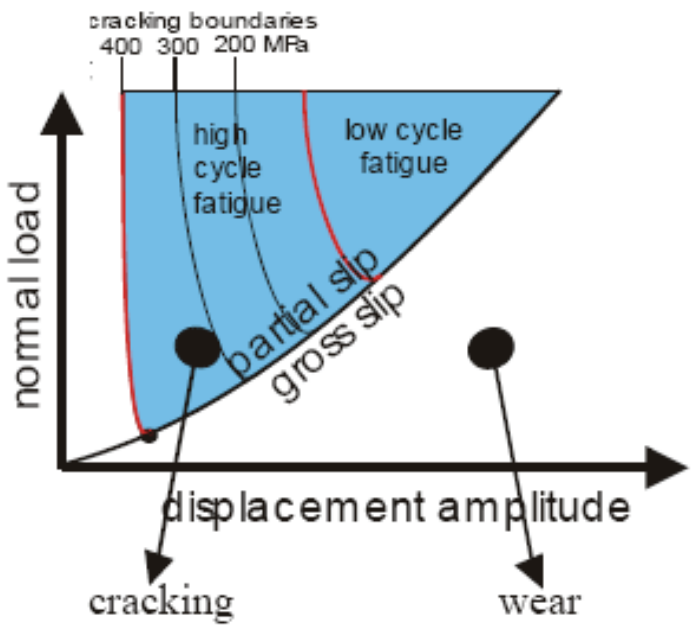

Fig. 1 A schematic plot of normal load against tangential displacement identifying the partial and gross slip regions. The effect of different external loads on the cracking boundary is also shown

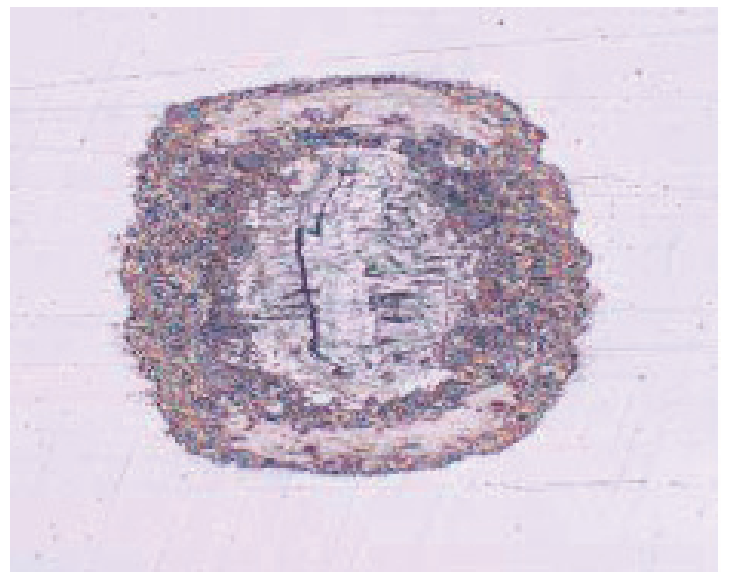

Fig. 2 A photograph showing a qualitative example of a ball-on-flat contact area that has suffered fretting damage with a visible micro-crack

Fretting fatigue has earlier been studied in detail for single contacts. The present work focus on how to put the models into a bearing simulation framework using BEAST. This enables the use of the fretting fatigue models to predict failures, as well as to provide a platform to develop the fretting fatigue models further.

The focus of this work is on dealing with implementation issues that arise when the model is moved to a transient bearing framework.

\section{Modelling fretting fatigue}

Fretting fatigue is a possible failure mode of rolling bearings, as well as for other machine elements with contacts. For a rotating bearing it normally can occur in the interfaces between outer ring/housing, and inner ring/shaft. For a non-rotating bearing subjected to small motion due to vibration, it can occur in the concentrated rolling element/ring contacts as well (mainly related to false-brinelling).

The fretting fatigue model predicts the risk of crack initiation. The Dang Van multi-axial fatigue criterion is utilized to predict the risk $D$ for crack initiation. The Dang Van algorithm used here is presented in ${ }^{2)}$.

The microscopic stress state considered in this crack initiation scheme is of mesoscopic scale, in other words, the order of magnitude considered is of the size of a material grain. The microscopic stress tensor $(\sigma)$ is derived from the macroscopic stress tensor $(\Sigma)$ following the relationship:

$$
\sigma_{i, j}(x, y, z, t)=\Sigma_{i, j}(x, y, z, t)+\rho_{i, j}^{*}(x, y, z)
$$

where $\rho_{i, j}$ is the local residual stress field. The spatial coordinates are $x, y$ and $z$ while $t$ represents time. According to Melan's theorem when shakedown occurs it reaches a time independent state $\rho_{i, j}^{*}$. Assuming a combined isotropic and kinematic hardening material, the stabilized residual stress field is: $\rho_{i, j}^{*}=-\operatorname{dev}\left(\bar{\Sigma}_{i, j}\right)$ where $\operatorname{dev}()$ is the deviatoric part of the tensor $\bar{\Sigma}_{i, j}$ which is the mean of $\Sigma$. The characteristic relationship of the Dang Van critical plane approach is then:

$$
\max _{\vec{n}}\left\{\max _{t}\left[\tau(\vec{n}, t)+\alpha \sigma_{\mathrm{H}}(t)\right]\right\}
$$

Where $\sigma_{\mathrm{H}}$ is the hydrostatic stress, $\alpha=\left(6 t_{-1}-3 f_{-1}\right) / 3 f_{-1}$ and $\beta=2 t_{-1}$. Hence, the model only takes into consideration crack mode II, since the plane of normal unit $\vec{n}$ on which the maximum shear stress acts is considered. One can simplify the determination of the criterion assuming Tresca's law is valid: $\quad \max _{\vec{n}}(\tau(\vec{n}, t))=\tau_{\text {Tresca }}(t)$ and the plane considered is such that $\vec{n}=\frac{1}{\sqrt{2}}\left(\frac{\vec{\sigma}_{i}}{\left\|\vec{\sigma}_{i}\right\|}+\frac{\vec{\sigma}_{j}}{\left\|\vec{\sigma}_{j}\right\|}\right)$. Hence one has:

$$
D=\max _{t}\left[\frac{\tau_{\text {Tresca }}(t)}{\beta-\alpha \sigma_{\mathrm{H}}(t)}\right]
$$

A value of $D \geq 1$ means that we have crack initiation, and $D=1$ means that there is no risk at all for fretting fatigue. The input to this calculation is transient stresses at the surface ("at" means here on and just under the surface), and the evolution of a residual stress field from plastic deformation due to the stresses above. The fretting material data used are $\left(f_{-1}=\sigma_{\mathrm{d}}=690 \mathrm{MPa}, t_{-1}=\right.$ $\left.\tau_{\mathrm{d}}=428 \mathrm{MPa}, E=428, v=0.3\right)$. 


\subsection{Stress and deformation calculation}

In an ideal world one can model, e.g., with FEM, the whole application in such fine detail that one can resolve details in concentrated contacts as well the global behaviour in one model. However, this is not practical from a simulation point of view. In BEAST we solve this issue by separating the contact deformation from the structural deformation, i.e., the flexible bodies act as boundary condition to the contact model. We employ reduced models (global shape or CMS-Component Modal Synthesis) for the structural flexibility, if it is needed.

As in any use of superimposing techniques and use of different scales, care has to be taken that the assumptions hold and that effects are not added twice.

The sub-surface contact stress at the surface, i.e., just under the surface, is implemented by sub-surface stress calculation in an elastic half-space based on Boussinesq-Cerruti integral, see for example ${ }^{5)}$ for derivation of influence functions. To get sufficient speed in the stress calculations we utilize MultiLevel MultiIntegration ${ }^{4}$. In a multi-body simulation tool like BEAST the state variables (positions and velocities) together with the geometry for each body is used to compute the pressure and traction fields in a contact. The pressure and traction fields are then used as input for the sub-surface stress calculations. A schematic diagram is shown in Fig. 3.

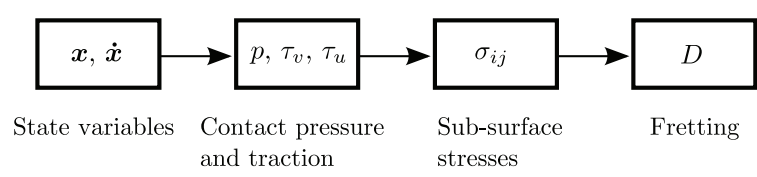

Fig. 3 The calculation flow for contact stresses and fretting calculations in BEAST

\subsection{Contact surface shear stresses and stick-slip}

Generally when modelling fretting fatigue, the stick slip zones for incipient sliding is determined followed by calculating the associated stress distribution and the risk for fretting fatigue. The surface shear stresses build up elastically (stick) until the static friction limit is reached, and slip occurs. This gives the two different zones (stick and slip) inside a contact. We need to capture some of this behaviour but in a very simulation-friendly way.

Assume that most of the contact stresses/loads are carried by the asperities, then each point (asperity) on the surface is decoupled to some extent from the rest of the surface. In a conforming contact the pressure gradients are less severe; this also indicates less coupling between points over the surface.

These reasons, motivate a tryout of a simple bristle model ${ }^{5)}$, i.e., tangential elastic foundation model, for the two surface shear stress components. Of course, the applicability of such a model needs to be investigated, and coupling terms introduced if needed.

Inspired by the $\mathrm{LuGre}^{6}$ ) we formulate the rate of surface shear stress at each point on the surface as

$$
\dot{\tau}=k \cdot v_{\mathrm{S}}\left(1-\frac{\tau}{\tau_{\mathrm{S}}(t)}\right)
$$

where $v_{\mathrm{S}}$ is the relative slinding velocity, $k$ is a shear stiffness, and $\tau_{\mathrm{S}}$ is the complete "Stribeck" shear stress without any initial elastic behaviour. Note, that the $\tau_{\mathrm{S}}$ switches sign the same time as $v_{\mathrm{S}}$.

This equation is applied in each of the two parameter direction of the surface. The ODEs (ODE - Ordinary Differential Equation) are implemented with implicit Euler, which is unconditionally stable, and solved over the whole contact surface in each time step.

In Fig. 4, comparison is given between two different models giving the non-dimensional tangential force. The BEAST result is presented together with a numerical model from ${ }^{3)}$. The agreement between the two models is satisfactory keeping in mind the difference in complexity of how to determine shear forces.

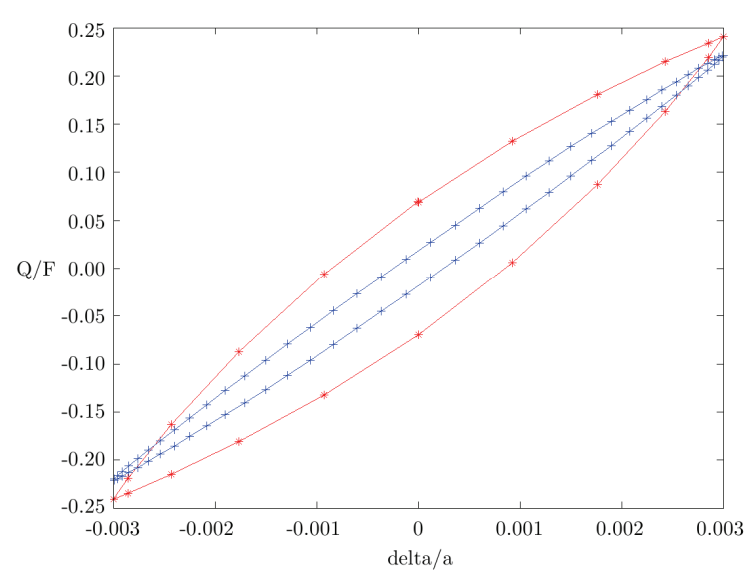

Fig. 4 Non-dimensional tangential force $(Q / F)$ as function of the non-dimensional tangential displacement (delta/a) with two different models; Kalker ${ }^{3)}$ (blue line) and BEAST (red line). The tangential force $(Q)$ is made non-dimensional regarding the normal load $(F)$ and the displacement (delta) is made non-dimensional regarding the semi-axis $(a)$ of the Hertzian contact

\subsection{Stabilized local residual stress}

The Dang Van model includes residual stresses due to the deformation history. For a cyclic loading, the residual stresses can be estimated with a mean value of the stresses $^{2)}$. In a transient simulation framework, this has no meaning. We have instead used a running average. It has the mean value as asymptote.

$$
\begin{gathered}
\bar{x}_{n}=\frac{\sum_{i}^{n} x_{i}}{n}=\frac{\sum_{i}^{n-1} x_{i}}{n-1} \cdot \frac{n-1}{n}+\frac{x_{n}}{n}= \\
\bar{x}_{n-1} \cdot \frac{n-1}{n}+\frac{x_{n}}{n}
\end{gathered}
$$


The notation is as follows:

$n$ the current step

$x_{i}, x_{n-1}, x_{n}$ the value at step $i, n-1$ and $n$ respectively $\bar{x}_{n-1}, \bar{x}_{n}$ the mean value after $n-1$ and $n$ respectively

This can be improved upon by using the proper hardening/plasticity models and solving those equations over time. Thus, we obtain a generic transient formulation and do not need to assume anything about the loading.

\section{Experimental verification}

All the experiments were carried on a fretting test rig, see Fig. 5. The experiments were conducted with AISI 52100 bearing steel balls in contact with steel plates under fretting loading conditions, implying a constant normal preload $F$ and a relative oscillating tangential motion with amplitude $c$ and frequency $f$. The fretting parameters (mainly, $F$ and $c$ ) were controlled to provide the partial slip regime (which is known to be the most dangerous regarding the risk of crack initiation) throughout the experiments. In some experiments the plates were also pre-stressed by applying a bending force $N$ in the middle of the bottom facet, which caused a maximum displacement $\delta$ in the middle of the flat.

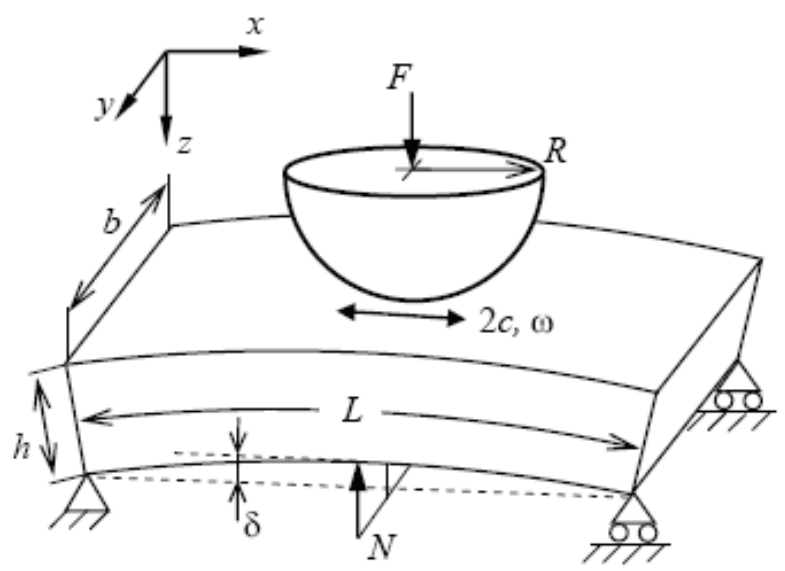

Fig. 5 Experimental set-up

The balls and the plates were produced of the same bearing steel. The contacting facets of the plates were polished in order to reduce the effect of the surface roughness. Each experiment was stopped after visible cracks had appeared within the plate (usually very close to the leading and trailing edges of the contact area), or after maximum 72000 fretting cycles. If no visible cracks had appeared after this number of cycles, the loading conditions were assumed to be safe. Typical fretting cracks within the plate are shown in Fig. 6.

Figure 7 represents the shear force loops obtained with a detailed numerical model (solid lines), in comparison with the simplified analytical solution of Johnson $^{5)}$ (dashed line), and experimental data for the same loading conditions. The detailed numerical model
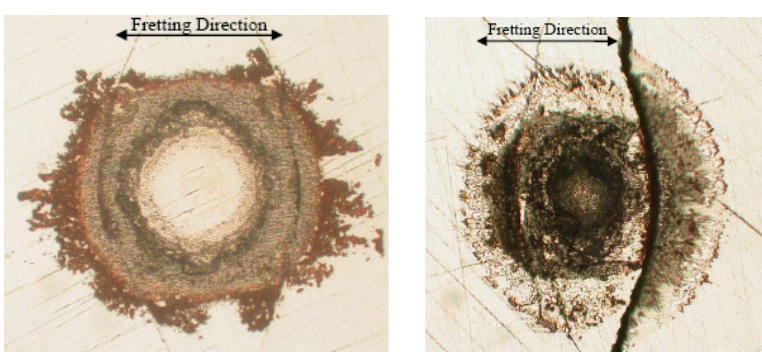

Fig. 6 Typical fretting cracks within the plate for $F=$ $122 \mathrm{~N}$ and $c=4$ : (a) Two shallow cracks for $\sigma=$ $430 \mathrm{MPa}$; (b) a complete breakage of the plate for $\sigma=1270 \mathrm{MPa}$. The fretting direction is shown by the black arrows.

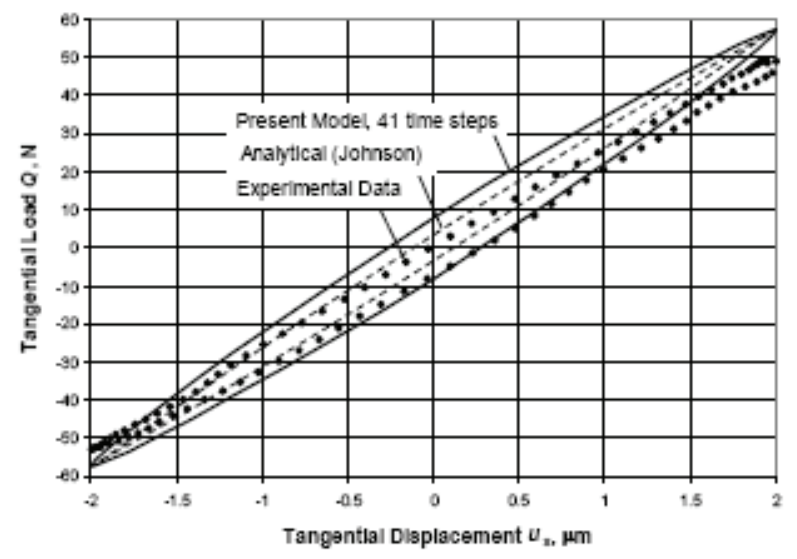

Fig. 7 Comparison of shear force loops, obtained in different ways, for the same loading conditions: a detailed numerical model, Johnsons analytical solution $^{5)}$, and experimental data

agrees satisfactory both with the analytical solution and with the experimental data.

Figure 8 shows comparison between the predictions of the numerical model and the experimental data concerning the boundary between the safe and unsafe fretting risk regions. The red dots represent experiments, where visible cracks within the plate were observed before 72000 cycles. The blue dots correspond to those cases, where no visible cracks were found (though, there could be invisible microcracks). The model predictions are shown by the thick solid line, while the trend line of the experimental data is shown by the thin line. The dashed line shows the elastic limit with respect to the normal load.

As can be seen from Fig. 8, there exists a good qualitative agreement between the model and experiments: the shapes of the boundaries between the safe and unsafe regions are very similar. However, the theoretical boundaries lay somewhat beneath the experimental ones. This discrepancy could be probably attributed to the fact that the absence of visible cracks does not cancel the probability of invisible microdamages within the plate which might be caused by the fretting loading. Another source of the 


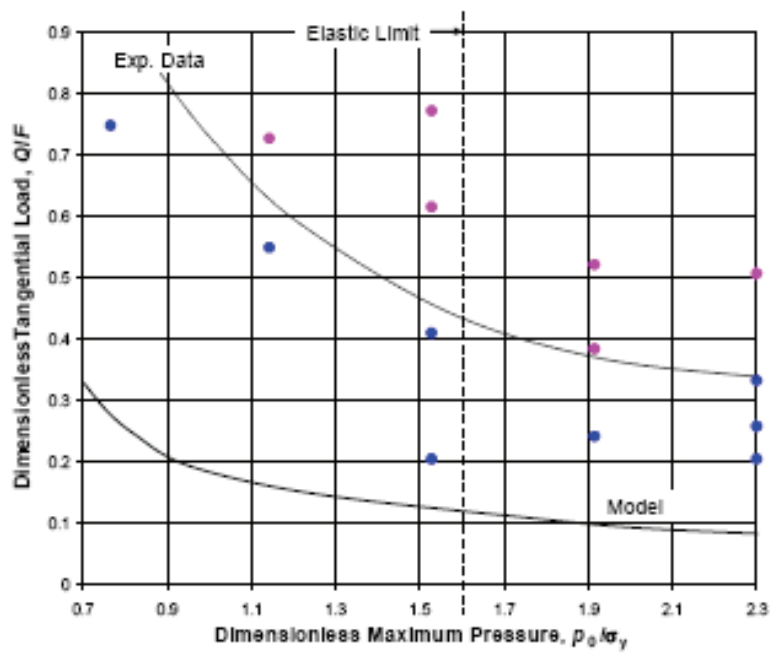

Fig. 8 The boundaries between the safe and unsafe regions - model vs. experiments. The red and blue dots correspond to a crack and no-crack, respectively, in the experimental data.

discrepancy is the elastic nature of the present numerical model, which does not take into account possible plastic deformations within the contact region. It should be noticed that even with entirely elastic normal loading, adding a tangential load will intensify the stress field in the contact zone and can cause appreciable plastic deformations.

\section{Experimental verification}

Here we study a concentrated point contact with a slow oscillating sliding motion. The combined curvature radius of both surfaces is $50 \mathrm{~mm}$ in both coordinate directions, the normal force is $1250 \mathrm{~N}$, the friction coefficient is 0.53 , the horizontal sliding amplitude is $2.241 \mu \mathrm{m}$, the semi-axis of the Hertzian contact is 747 $\mu \mathrm{m}$, the Young's modulus is $2.0 \times 10^{11} \mathrm{~Pa}$, and the Poisson's ratio is 0.3 .

Below there are two snapshots from the simulation, see Figures 9-10. They show a two-body model with the coefficient of friction $\tau / p$ on the lower body surface, and

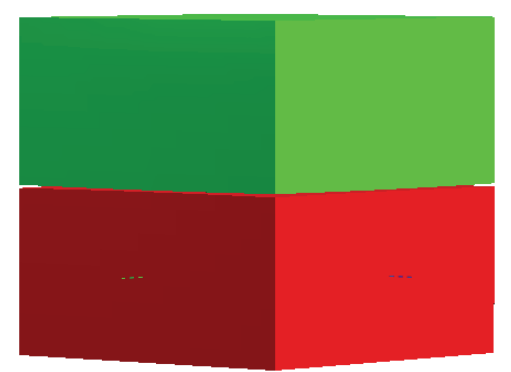

Fig. 9 A BEAST two-body model of a concentrated contact where each body has one curved surface
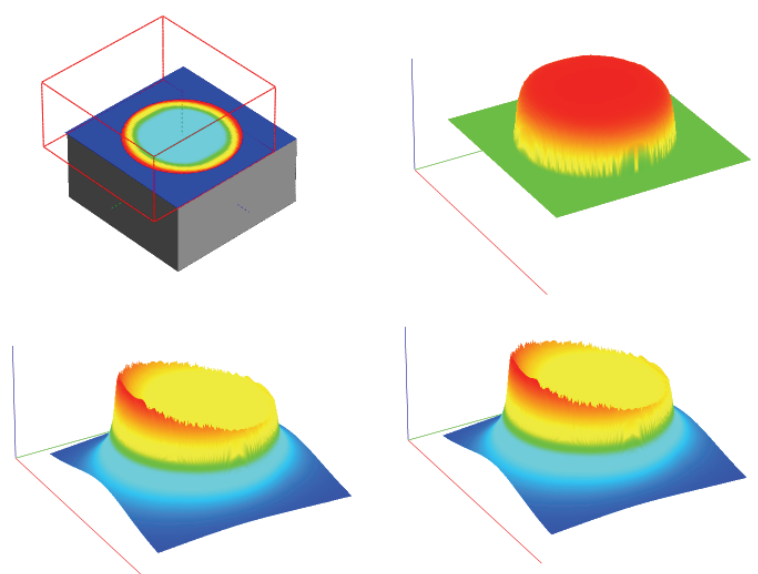

Fig. $10 \mathrm{~A}$ snapshot of qualitative results from a two-body model of a concentrated contact. The pictures show data from the lower body surface. They are; (upper left) the lower body with effective friction coefficient, (upper right) surface shear in sliding direction, (lower left) instantaneous fretting risk, (lower right) maximum fretting risk. The motion is magnified 300 times.
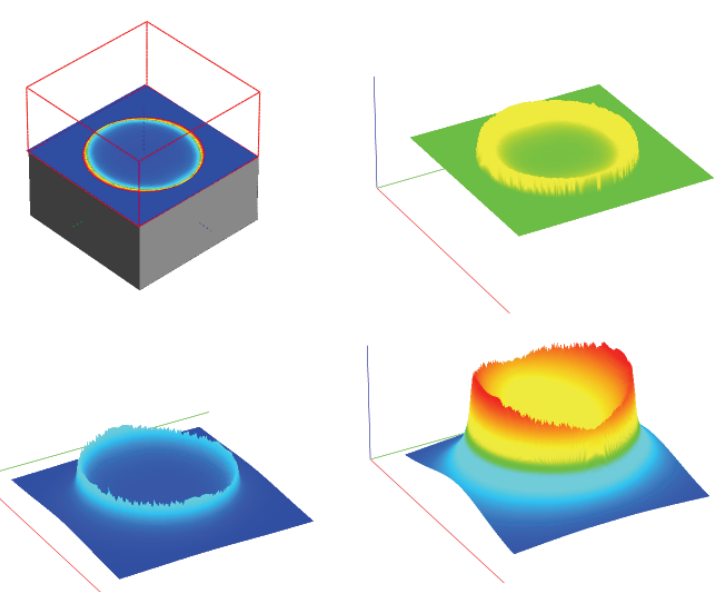

Fig. 11 A second snapshot of qualitative results from a two-body model of a concentrated contact. The pictures show data from the lower body surface. They are; (upper left) the lower body with effective friction coefficient, (upper right) surface shear in sliding direction, (lower left) instantaneous fretting risk, (lower right) maximum fretting risk. The motion is magnified 300 times.

in separate views surface shear in the sliding direction $\tau$, the instantaneous fretting risk, and the maximum fretting risk, all pictures representing qualitative results.

The stick/slip zones can be seen in the upper left figure in Fig. 10 - Fig. 11. The red color indicates a friction coefficient that corresponds to slip. 


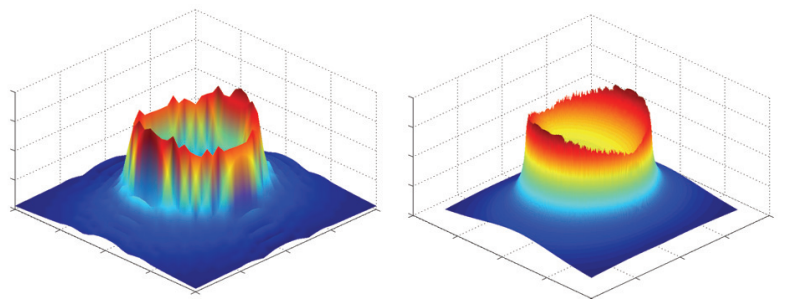

Fig. 12 Schematic views of the maximum fretting risk from a stand-alone code (left) and from BEAST (right)

The maximum Dang Van fretting risk parameter is around 0.8 in this case.

The same case has been run with a stand-alone code using a more detailed model for the shear stresses, see Kalker $^{3)}$ on the surface. The maximum fretting risk from both the detailed model, and the present BEAST simulation is shown in Fig. 12.

\section{Fretting of a deep grove ball bearing mounted in a ring}

Here we study an application case, a Deep Grove Ball Bearing (DGBB) mounted in a ring-shaped house, see Fig. 13. The outer ring is modelled as structural flexible, other bodies are stiff. However, all contacts are elastic. A displacement of $60 \mu \mathrm{m}$ is applied on the inner ring upwards vertically. The inner ring rotates with 3000 rpm. The friction coefficient on the house/outer ring interface is 0.11 , which is a typical value for a wet surface at zero sliding speed.

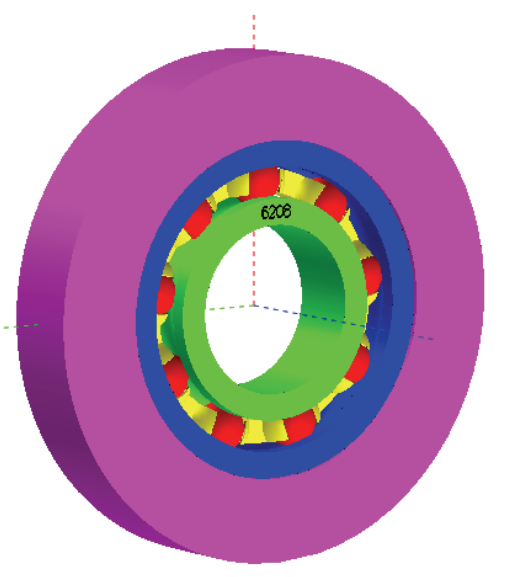

Fig. 13 A BEAST model of a deep grove ball bearing mounted in a ring-shaped house. The bearing is loaded by a vertical displacement of the inner ring upwards while keeping the house fixed.

In Fig. 14 a snapshot from the simulation is shown. It shows the DGBB with effective friction coefficient $\tau / p$ on in the outer ring surface, and in separate views the fretting risk, surface shear in circumferential direction $\tau$, and pressure $p$. It is easily seen from the figure that only the upper half of the circumference is subjected to load. The rest is unloaded and consequently has no fretting risk.

The ball pass effect can be seen on fretting risk, shear, and pressure. The behaviour is very dynamic. The deformation due to ball contact loading of the outer ring is shown in Fig. 15.

The Dang Van fretting risk parameter is less than 0.0029 in this case, which is very low.

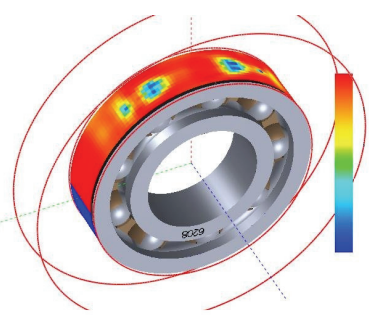

(c)

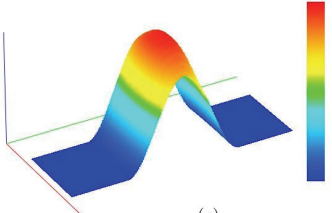

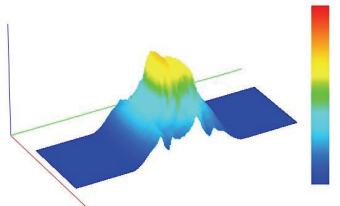

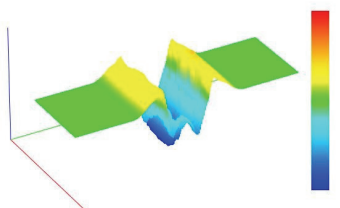

Fig. 14 A snapshot of results from a deep grove ball bearing mounted in a ring. The pictures show data from the outer ring interface. They are; (a) DGBB with effective friction coefficient, (b) fretting risk, (c) pressure, and (d) surface shear in circumferential direction.

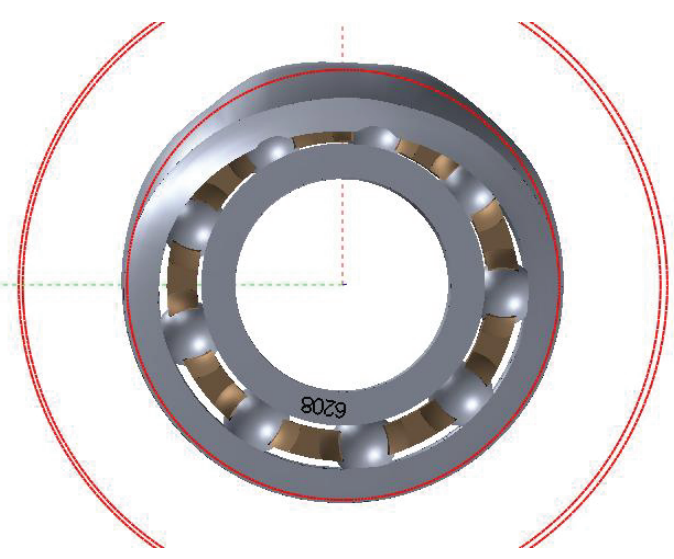

Fig. 15 A deep grove ball bearing mounted in a ring. The ball pass deformation is shown, it is magnified 4343 times.

\section{Discussion}

The use of a simple linear law to calculate the slip distribution in a contact combined with the failure criterion of Dang Van show a sufficiently fast methodology to predict fretting risk in transient situations in real machinery components. The fretting 
calculations using the implementation in BEAST are 2 3 order of magnitudes faster than the detailed numerical model.

Notice that the model for fretting as presented here is only verified for harmonic loading. It should be possible to implement a shakedown model for the stabilized local residual stress and proper rain-flow counting method to consider any non-harmonic load cycling.

Equally the model for elastic contact surface shear stresses and stick-slip works as intended and it is efficient from computation point of view. The main results regarding tangential force and maximum fretting risk (both magnitude and shape) agree with more accurate elastic shear model, see Kalker ${ }^{3}$. However, it is a simple shear model and it will investigated to make it more accurate without sacrificing simulation speed.

Further verification of the model for non-concentrated contacts is being done at the moment, results may be reported in a follow-up paper.

The fretting risk calculation model presented here, although simple, shows potential to build knowledge and develop solutions in practical industrial cases. Many improvements are possible (e.g. rain-flow, damage accumulation rule to account for life rather than only risk and fretting-wear considerations).

\section{Conclusion}

A computationally simple model to predict fretting risk in real machine components has been developed. The model uses the multiaxial fatigue failure criterion of Dang Van combined with a simple model to predict contact slip and tractions. Subsurface stresses are then calculated in time using MultiLevel Multi-Integration, the Dang Van risk parameter is then assessed. The model has been verified using a more sophisticated approach based on reference ${ }^{3)}$ and also the analytical model of ${ }^{5)}$, finally the model was compared versus experimental results. The comparison of the fretting loops for given normal load, fretting amplitude and friction coefficient reveals a good agreement between an analytical solution, numerical model and the experimental data. Concerning the prediction of the crack risk, a satisfactory agreement between the results of the numerical model and the experimental data was observed. Then the model was used in the calculation of fretting risk in the outer sit of a full rolling bearing, fretting risk is calculated as the rolling elements move. From the analysis carried out the following conclusions can be drawn:

1. A test implementation of fretting fatigue in a full component simulation tool (i.e. BEAST) has been created. It covers all types for contacts, e.g., both concentrated and conformal contacts. Two types of cases have been analysed, a concentrated point contact, and a deep grove ball bearing in a ring-shaped house. For the specific combinations of loads, materials, etc., they did not show risk for fretting fatigue.

2. The comparison between results from the detailed numerical model and results from BEAST shows satisfactory agreement, both for the shear loop and the fretting risk.

3. It has been shown that BEAST can be used as a test bench or framework for modelling physical phenomena on contacting surfaces, e.g., can be used as platform for further development of fretting models and evaluating fretting risk in rolling bearings.

\section{Acknowledgements}

The authors wish to thank SKF Group Product Technical Director, for his kind permission to publish this article.

\section{References}

[1] Stacke, L. E. and Fritzson, D., "Dynamic Behaviour of Rolling Bearings: Simulations and Experiments," Proc. Inst. Mech. Eng., Part J, Journal of Engineering Tribology, 215, 2001, 499-508.

[2] Dang-Van, K., Griveau, B. and Message, O., "On a New Multiaxial Fatigue Limit Criterion: Theory and Application," In M.W. Brown and K.J. Miller, editors, Biaxial and Multiaxial Fatigue (EGF 3), January Wiley Mechanical Engineering Publications, London, UK, ISBN 978-0-85298-669-1, 1989, 479-496.

[3] Kalker, J. J., "Three-Dimensional Elastic Bodies in Rolling Contact," Kluwer Academic, Dordrecht, 1990.

[4] Brandt, A. and Lubrecht, A. A., "Multilevel Matrix Multiplication and Fast Solution of Integral Equations," Journal of Computational Physics, 90, 1990, 348-370.

[5] Johnson, K. L., "Contact Mechanics," Cambridge University Press, Cambridge, 1987.

[6] Canudas, de W C., Olsson, H., Astrom, K. J. and Lischinsky, P. "A New Model for Control of Systems of Friction," IEEE Transactions on Automatic Control, 40, 3, 1995, 419-425. 